\title{
L'œuvre condéenne pour la jeunesse : entre échec littéraire et mises en scène de l'échec
}

https://doi.org/10.29173/af29434

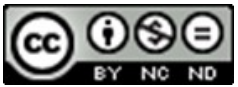

\section{Merveilles Mouloungui}

leoncia.mouloungui@univ-lorraine.fr

Université de Lorraine, France

Résumé. Le présent article s 'interroge sur la place de la littérature de jeunesse de Maryse Condé au sein de sa bibliographie générale, et dans la réception critique. La relative marginalisation de ces æuvres semble due à des facteurs spécifiques au secteur éditorial dans son ensemble, et à d'autres facteurs propres à la littérature francophone féminine, comme le manque de spécialistes dans le domaine et le manque d'instances de légitimation. Dans ce même corpus destiné, Maryse Condé déploie le plus souvent une écriture de l'échec, ce qui pourrait être interprété comme un écho de la faible visibilité institutionnelle des œuvres concernées. Quoi qu'il en soit, ces échecs dans la fiction sont certainement à mettre sur le compte des intentions réalistes d'un auteur qui ne cesse de renvoyer à la dureté d'un monde post-colonial auquel ses lecteurs seront ainsi préparés.

Mots clés: Maryse Condé; littérature de jeunesse; mixité éditoriale; échec littéraire; réalisme postcolonial.

Abstract. This article examines the place of Maryse Condé's children's literature within her general bibliography, and in critical reception. The relative marginalization of these works seems to be due to factors specific to the publishing sector as a whole, and to other factors specific to Francophone women's literature, such as the lack of specialists in the field and the lack of legitimating bodies. In this same corpus, Maryse Condé most often writes about failure, which could be interpreted as an echo of the low institutional visibility of the works concerned. In any case, these failures in fiction can certainly be attributed to the realistic intentions of an author who constantly refers to the harshness of a post-colonial world for which her readers will thus be prepared.

Keywords: Maryse Condé; children's literature; editorial mix; literary failure; postcolonial realism. 
If we judge children's books (even if we do it unconsciously) by the same value systems as we use for adult books-where they are bound by definition to emerge as lesser - then we give ourselves unnecessary problems. To say that, for example, Judy Blume is not as good as Jane Austen, is likes saying that this apple is an inadequate orange because it is green, and that oranges are innately superior anyway.

Peter Hunt (3-4)

$\mathrm{L}$ a « grande » littérature de Maryse Condé connaît un succès indéniable ${ }^{1}$ dans le vaste champ de la littérature francophone et, depuis 2007, de la littérature-monde en français. Parallèlement, Condé est aussi auteure d'un corpus littéraire destiné à la jeunesse, mais, malheureusement, celui-ci a été moins bien reçu par la critique du fait d'un manque de légitimité de cette littérature. En effet, « l'activité littéraire, en particulier, dans son déploiement et dans sa pratique, reste intimement liée à des instances idéologiques, culturelles, économiques, etc., qui en assurent la circulation et surtout la valeur sur le marché des biens symboliques » (Amedegnato et al. 11). Or, l'institution littéraire peine encore à faire une place à la littérature de jeunesse, jugée marginale. Si, comme l'indique Christophe Bertiau, " l'histoire de la littérature est avant tout une histoire du succès [qui] ne retient que les œuvres qu'elle juge dignes de considération [et que] son silence signifie l'échec » (1), on peut considérer l'œuvre condéenne destinée à la jeunesse comme un échec littéraire. Or, une analyse de ces textes révèle une écriture centrée sur les défaites. Simple coïncidence? Sûrement, étant donné qu'une bonne partie de sa production littéraire a en commun, parmi plusieurs éléments esthétiques, cette poétique de l'échec. En effet, qu'elle s'adresse aux adultes ou aux enfants, l'écrivaine organise ses intrigues romanesques autour d'événements « troublants » caractérisés par des tonalités dramatiques, pathétiques et tragiques. Depuis son premier roman destiné au jeune public, Haïti chérie (1987) qui se clôt sur l'échec de la traversée et la mort de l'héroïne, jusqu'à son dernier roman La Belle et la Bête : une version guadeloupéenne (2013) qui s'achève sur l'échec du mariage des deux personnages, l'auteure nous offre des univers marqués par l'échec ${ }^{2}$. On peut ainsi se demander s'il existe des rapports de cause à effet entre l'échec de cette littérature et les mises en scène de l'échec qui la caractérisent.

\section{LITTÉRATURE DE JEUNESSE vs LITTÉRATURE POUR ADULTES}

Maryse Condé a collaboré plusieurs fois avec le magazine Je bouquine qui l'a sollicitée pour publier à l'intention des adolescents. Cela a donné les récits suivants : Haïti chérie, Victor et les barricades (1987), Savannah blues (2004) et Chiens fous dans la brousse (2006), dont les intrigues se déroulent respectivement en Haïti, en Guadeloupe, au Mali et en Amérique, soit aux quatre coins du monde ou presque. En dehors de cette fructueuse collaboration, elle a publié d'autres ouvrages dans le même genre ;

\footnotetext{
${ }^{1}$ Largement étudiée et traduite dans plusieurs langues, elle a été, plusieurs fois, primée par des instances littéraires caribéennes (Prix Carbet de la Caraïbe, pour Desirada, par exemple) et mondiales (Prix Puterbaugh, pour l'ensemble de son œuvre, Grand prix du roman métis pour En attendant la montée des eaux, entre autres).

${ }^{2}$ Le constat est le même en ce qui concerne sa littérature pour les adultes, de ses premières œuvres (Dieu nous l'a donné, Héremakhonon et Une saison à Rihata) qui problématisent l'échec du retour identitaire à sa dernière œuvre (Le fabuleux et triste destin d'Ivan et Ivana) qui se clôt sur la mort des personnages centraux.
} 
il s'agit de Hugo le terrible (1991), La planète Orbis ${ }^{3}$ (2002), À la courbe du Joliba ${ }^{4}$ (2006), Conte cruel (2009) et La Belle et la Bête : une version guadeloupéenne. Maryse Condé compte ainsi neuf textes écrits à destination du jeune public, auxquels on pourrait ajouter la seconde version de sa biographie : Le cœur à rire et à pleurer : contes vrais de mon enfance (2013), dont l'édition destinée aux personnes de niveau de français B2 semble s'adresser à un public adolescent.

Cette production relativement foisonnante, qui compte à la fois des contes, des romans, un album et un récit de science-fiction, confère à Maryse Condé une nouvelle image, celle d'une écrivaine pour la jeunesse, bien que ce corpus semble marginalisé au sein de sa bibliographie générale. L'auteure ellemême entretient des liens différents avec les deux secteurs : si elle arrive à la littérature pour les adultes par passion, son entrée en littérature de jeunesse s'explique par le besoin de répondre à une commande, comme en témoignent les extraits suivants de deux entretiens distincts. Le premier explique son entrée en littérature pour les adultes :

— Maryse, comment es-tu venue à la littérature ?

Eh bien, je crois que j'ai toujours écrit. Je me rappelle avoir écrit pour l'anniversaire de ma mère une sorte de pièce de théâtre en un acte dont elle était l'unique personnage. C'était elle qui parlait, qui répondait, qui agissait. [...] Quand j'étais étudiante, j'écrivais des comptes rendus de livres dans une revue d'étudiants qui s'appelait, je crois Alizé, et ensuite, en Afrique, j'ai pris des notes pour écrire plus tard. C'est sur ces noteslà que j'ai travaillé Hérémakhonon et Une saison à Rihata. (Pfaff 55)

Et le second, quant à lui, met en avant les raisons de sa mixité éditoriale :

Cécile Lebon : Maryse Condé, dans votre bibliographie très importante, on trouve des romans pour les jeunes. Est-ce que ces livres écrits pour les adolescents sont nés de la demande d'un éditeur, ou d'un désir - la volonté de dire quelque chose à un jeune public, de réagir à une situation politique, désir littéraire de s'essayer à un nouveau genre... ?

Maryse Condé : Quand j'écris pour les jeunes, des enfants, je réponds généralement à une demande. D'abord, mes petits enfants se sont plaints que je n'écrive jamais pour eux, que les livres soient toujours dédiés à leur mère, tantes ou grand-père. (Condé 38)

Il suffit d'observer les deux réponses de l'auteure pour comprendre que cette dernière procède par « circulation descendante ${ }^{5}$ et que l'initiative de s'engager dans la littérature de jeunesse n'a pas été spontanée.

\section{PRÉSENCE DE L’AUTEURE DANS LES CHAMPS ÉDITORIAUX}

\footnotetext{
${ }^{3}$ La Planète Orbis est le roman de science-fiction de l'auteure, illustré par Letizia Galli.

${ }^{4}$ Publié chez Grasset-Jeunesse, À la courbe du Joliba est le seul album de Maryse Condé ; il est illustré par Letizia Galli et adressé au jeune public à partir de 8ans.

5 «La mobilité des auteurs s'opère dans les deux sens : de nombreuses plumes consacrées dans le champ de la littérature générale viennent publier dans des collections destinées aux adolescents (je parlerai de "circulation descendante"), et [des] auteurs établis dans le champ de la littérature pour la jeunesse font leur entrée en littérature générale (on parlera de "circulation ascendante"). Je précise à toute fin utile que la "descente" et l'“ascension" n'ont ici aucune valeur appréciative, mais se réfèrent uniquement à l'âge des lecteurs. » (Delbrassine 164).
}

Alternative francophone

https://journals.library.ualberta.ca/af/index.php/af 
La présence de Maryse Condé dans le champ éditorial pour la jeunesse se résume à quelques apparitions et elle a donné lieu à relativement peu de mentions dans les espaces de diffusion spécialisés. Les facteurs déterminants la visibilité dans les deux secteurs sont en effet différents, ainsi que le démontrent certaines revues spécialisées comme Takam Tikou : la revue des livres pour enfants. D’ailleurs, dans le numéro 38, qui propose un dossier intitulé « La Caraïbe et le livre de jeunesse », on retrouve un entretien de l'écrivaine avec Cécile Lebon. Bien sûr, puisqu'il s'agit d'une revue consacrée à la littérature de jeunesse, l'échange porte sur cette production littéraire, précisément sur les ouvrages Haïti chérie, Victor et les barricades, Hugo le terrible et La Planète Orbis, déjà publiés à ce moment. On trouve aussi une notice consacrée à Maryse Condé dans le dictionnaire du livre de jeunesse codirigé par Isabelle Nières-Chevrel et Jean Perrot (2013). Outre ces mentions, l'auteure a plusieurs fois été invitée dans des écoles pour parler de ses ouvrages ou pour animer des ateliers d'écriture, des pratiques très courantes dans le champ de la littérature pour la jeunesse où le contact avec le lecteur dans des lieux d'apprentissage et, plus largement, de culture est d'une importance capitale pour la diffusion du livre et la sensibilisation à la lecture.

Ces activités peu nombreuses révèlent la faible implication de l'auteure dans ledit champ, où sa visibilité reste faible. Il n'en va pas de même dans le-secteur de la littérature générale où elle a été distinguée par le prix Nobel Alternatif en 2018, après avoir obtenu le Grand Prix littéraire de la Femme pour Moi, Tituba sorcière... noire de Salem, le prix Anaïs-Ségalas de l'Académie française pour La Vie scélérate, le Prix Marguerite-Yourcenar pour Le Cour à rire et à pleurer et le Grand prix du roman métis pour En attendant la montée des eaux, pour ne citer que ces récompenses. Ce succès non contesté a couronné des œuvres qui s'insèrent très bien dans les circuits de légitimation du secteur adulte. Du reste, sa littérature de jeunesse doit son existence à celle qu'elle a destinée aux adultes, puisque si Bayard Jeunesse lui a proposé une collaboration, c'est parce que l'écrivaine bénéficiait déjà d'un capital symbolique indéniable.

On note tout de même une volonté de l'auteure d'intégrer la bibliothèque pour les enfants d'autant qu'elle a publié un album et un récit de science-fiction, des genres très appréciés par les jeunes aujourd'hui. À propos du genre de l'album, Isabelle Nières-Chevrel rappelle qu'il s'agit de « la grande invention de la littérature de jeunesse » (119); de ce fait, on ne saurait s'étonner du fait que ce soit la couverture de l'album À la courbe du Joliba qui a été retenue pour illustrer la notice consacrée à Maryse Condé dans $L e$ dictionnaire du livre de jeunesse cité plus haut, notice qui atteste de sa reconnaissance relative dans le secteur jeunesse.

Cette faible visibilité n'est pas propre à l'œuvre condéenne dans la mesure où ses confrères Dany Laferrière, Patrick Chamoiseau, Gisèle Pineau, etc., sont rarement interrogés sur leurs œuvres pour la jeunesse, qui semblent elles aussi demeurer à la marge de leurs productions respectives. Bien qu'il s'agisse d'auteurs reconnus, le statut marginal de cette littérature demeure en effet un frein pour sa visibilité. De façon générale, la littérature de jeunesse ne bénéficie pas d'un rayonnement important et les auteurs à la recherche d'une reconnaissance se tournent souvent vers le secteur pour adultes. C'est ce qui semble expliquer, selon Kodjo Attikpoé, la forte présence des auteurs féminins dans le secteur pour la jeunesse : « la méfiance des hommes s'explique sans doute par leur perception de la littérature d'enfance et de jeunesse, souvent considérée comme une littérature de seconde zone, un genre peu porteur. Tout porte à croire que les écrivains hommes sont davantage en quête de reconnaissance, particulièrement dans le giron de la Francophonie. » (152)

En somme, si Maryse Condé est moins présente dans le secteur pour enfants que dans le secteur général, son œuvre dédiée l'est encore plus, car elle peine à intégrer les études littéraires, notamment au niveau de la critique universitaire.

\section{FAIBLE RÉCEPTION CRITIQUE}


Dans le cas de la production francophone, où la production pour les adultes souffre déjà d'un manque d'institutions, la situation de la littérature de jeunesse est encore moins avantageuse, malgré la naissance de plusieurs maisons d'édition spécialisées. En effet, l'insuffisance (et parfois l'absence) des politiques du livre ainsi que le taux d'alphabétisation dans certains secteurs de la société qui seraient plus favorables à la lecture de cette littérature de jeunesse francophone, parfois trop élevé freinent le développement de ce secteur éditorial encore fragile. Dans l'espace caribéen précisément, la situation de l'édition pour la jeunesse n'est pas vraiment distincte de celle qui est observée dans les autres pays du Sud, puisque, si elle parvient difficilement à s'exporter, elle ne séduit pas non plus - en tous cas pas comme elle devrait - les populations locales qui manquent encore de culture livresque. Dans un entretien, le professeur et écrivain Max Jeanne observe qu'entre 1980 et 2007, il y a « une évolution qualitative [du] nombre d'écrivains confirmés [qui] se sont investis dans cette littérature de jeunesse [...] » et que « le lectorat est mieux formé et informé » (Jeanne 333); mais il n'hésite pas non plus à souligner les difficultés auxquelles elle se trouve confrontée :

À l'évidence, cette littérature peut encore se développer. Mais cela suppose un partenariat constructif entre les auteurs (encore trop frileux) et les écoles. Et aussi une politique du livre de la lecture volontariste. La littérature reste encore le parent pauvre pour nombre de décideurs politiques plutôt chiches en matière de subvention (pourquoi pas des commandes aussi de la part des élus à tel ou tel écrivain ?) Cette littérature gagnerait à s'articuler autour de certains temps forts pérennes : salon du livre de Point-à-Pitre qui n'a pas eu lieu depuis deux ans, Festival du théâtre des Abîmes [...], prix littéraires. (Jeanne 335)

Ainsi, la littérature francophone pour la jeunesse semble avoir un double statut marginal, dans la mesure où elle appartient à la littérature francophone et relève du secteur de la littérature pour la jeunesse.

Cela étant, Maryse Condé est assurément l'une des auteures pour la jeunesse les plus lues en Guadeloupe et sa production destinée au jeune lectorat a fait l'objet de quelques études, notamment Haïti chérie $e^{6}$ qui a connu un succès auprès des universitaires, et Chiens fous dans la brousse ${ }^{7}$ qui a fait l'objet de plusieurs réflexions concernant l'écriture de l'esclavage et les liens que ce récit entretient avec le roman à succès de l'auteure : Ségou : les murailles de terre. On note aussi que l'ouvrage de Jean Perrot, Mondialisation et littérature de jeunesse (2008) s'intéresse à l'album À la courbe du Joliba, et plusieurs articles qui interrogent de façon générale la production pour la jeunesse de Maryse Condé soit en lien avec une thématique précise, soit simplement dans le cas d'une étude sur la littérature de jeunesse en Caraïbe (Bonnet 2017).

Il n'en reste pas moins que la littérature condéenne pour la jeunesse bénéficie d'une très faible réception comparativement à celle dont bénéficie son corpus pour les adultes, maintes fois analysé dans le cadre de thèses de doctorats, d'articles scientifiques, de colloques et d'ouvrages collectifs. Cette différence s'explique d'une part, par la négligence relative dont la littérature de jeunesse fait l'objet et, d'autre part, par le faible nombre des chercheurs intéressés par la littérature de jeunesse en général et par la littérature francophone de jeunesse en particulier, un domaine d'études en plein essor, car cette littérature de

\footnotetext{
${ }^{6}$ Assurément, le roman jeunesse le plus lu de Maryse Condé.

${ }^{7}$ Ce roman a fait l'objet de plusieurs articles scientifiques : «Littérature de jeunesse et esclavage : Chiens fous dans la brousse de Maryse Condé » (Fort), « De Ségou à Chiens fous dans la brousse : un exemple d'autoréécriture chez Maryse Condé » (Franchini).
}

Alternative francophone

https://journals.library.ualberta.ca/af/index.php/af 
jeunesse francophone est doublement marginalisée (d'abord comme francophone, ensuite comme «jeunesse »).

Dans l'introduction de l'ouvrage La littérature de jeunesse en question(s), Nathalie Prince déplore le fait que, bien qu'elle fasse partie des littératures les plus lues, la littérature de jeunesse continue d'être perçue comme une littérature
« facile », de qualité souvent médiocre, fondée sur des récits et des textes simples - sinon simplistes - qui ne méritent pas l'attention du public critique et universitaire. Entre le lectorat jeune, vert, plutôt naïf et sans subtilité - dit-on - de la littérature de jeunesse, et les exigences universitaires, l'écart est peut-être tel que leurs relations restent difficiles, ce qui explique sans doute - et nous le regrettons -, que la littérature de jeunesse demeure peu étudiée et encore mal considérée des compétences universitaires, même si certains professeurs ont consacré l'essentiel de leurs recherches à la défense et illustration de la littérature de jeunesse et ont brillamment ouvert la voie. (Prince 9)

Ce constat mitigé vaut évidemment pour la majorité des productions littéraires bien que quelques cas issus de la littérature mondiale fassent exception à cette réalité : L'Âne culotte (1937) d'Henri Bosco, Les Contes du chat perché (1934) de Marcel Aymé, Le Petit Prince (1943) d'Antoine de Saint-Exupéry, La Gloire de mon père (1957) et Le Château de ma mère (1957) de Marcel Pagnol ainsi que Vendredi ou la vie sauvage (1971) de Michel Tournier, sont quelques exemples de romans français pour la jeunesse qui ont bénéficié d'une bonne réception auprès du public universitaire, souvent en partie grâce à la célébrité de leurs auteurs déjà reconnus dans le champ pour les adultes. Raison de plus pour s'étonner du fait que la production destinée à la jeunesse d'une auteure aussi connue que Maryse Condé soit aussi peu reconnue, malgré ses nombreuses collaborations avec des éditeurs réputés (Bayard Jeunesse, Grasset Jeunesse, Larousse). Toutefois, c'est le cas pour la majorité des auteurs francophones du Sud qui peuvent être qualifiés d'écrivains « mixtes », comme Ahmadou Kourouma, Léonora Miano, Tahar Ben Jelloun, Dany Laferrière ou Edwige Danticat.

Au-delà de la marginalité de la littérature de jeunesse, une autre raison pourrait justifier la faible réception des œuvres destinées à la jeunesse par Maryse Condé. En effet, comme nous l'avons vu, celle-ci procède par « circulation descendante », après s'être positionnée dans le secteur de la littérature générale; dès lors, on peut supposer que son image demeure attachée à un type de production (pour les adultes) et que la conscience collective a, par conséquent, des difficultés à l'intégrer comme productrice d'un second type de littérature.

Ce relatif échec de l'œuvre condéenne pour la jeunesse conduit à s'interroger sur la textualité même des œuvres concernées. On peut notamment se demander si, pour une écrivaine reconnue pour son caractère « rebelle », il n'est pas assez difficile d'intérioriser les règles d'un champ tel que l'édition de jeunesse où le ton est généralement plus optimiste.

\section{L’ÉCHEC DANS LES ROMANS}

Plusieurs romans de Maryse Condé, tous secteurs confondus, reposent sur des intrigues dont la tournure dramatique et parfois même tragique peut émouvoir profondément le lecteur. Dès ses premières œuvres, Maryse Condé conduit ses hérö̈nes à l'échec : dans Hérémakhonon et Une saison à Rihata, elle met en scène des personnages qui vont en Afrique à la recherche de leur identité, mais qui finissent par se perdre 
dans le contexte politique post-colonial ${ }^{8}$ des pays concernés, où elles déchantent à la fois à propos de la société africaine et de l'amour. Toutefois, lorsque Françoise Pfaff demande à Maryse Condé s'il s'agit là de romans de l'échec, cette dernière répond en ces termes : «Je ne sais pas si c'est un roman de l'échec. Veronica était venue chercher une chose qu'elle n'a pas trouvée. Mais elle a trouvé une autre chose qu'elle n'était pas venue chercher. Il n'y a pas d'échec total, il n'y a pas de réussite totale et on trouve toujours quelque chose de valide dans toutes les démarches qu'on fait. » (Pfaff 63) Ces itinéraires fictionnels ressemblent beaucoup à celui de l'écrivaine elle-même dont le séjour en Afrique aux côtés de Mamadou Condé, son ex-époux, n'avait pas été une réussite. Cette dimension autobiographique, d'ailleurs très bien assumée par l'auteure, traverse l'ensemble de ses œuvres puisque, outre ces deux romans, la majorité de ceux qui suivent reproduisent pratiquement le même schéma narratif ; en effet, il est généralement question de protagonistes faibles et opprimés, évoluant dans des sociétés inégalitaires. Ainsi, qu'il s'agisse des romans écrits pour les adultes comme Moi Tituba sorcière ... noire de Salem, Le fabuleux et triste destin d'Ivan et Ivana, ou des textes destinés au jeune lectorat comme Haïti chérie, Chiens fous dans la brousse ou La Belle et la bête : une version guadeloupéenne, les constructions narratives semblent toujours mener les personnages à des formes d'échec ; d'un échec, certes, on peut tirer une leçon, ou un renouvellement de la quête initiale : c'est le propos d'adulte que tient l'auteur à ce sujet, et c'est aussi l'art de la nuance à laquelle se prête fort bien le genre romanesque pour adulte.

\section{DES SCHÉMAS NARRATIFS TORTUEUX}

On s'étonne par ailleurs de la structure complexe des œuvres condéennes, qui diffère en cela de la plupart des histoires que l'on raconte généralement aux enfants, lesquelles répondent à une progression bien précise : situation initiale - élément perturbateur - péripéties - résolution des péripéties - situation finale, le plus souvent heureuse. Or, si on s'en tient à Haïti chérie, Chiens fous dans la brousse et La Belle et la Bête, on ne peut pas dire que l'auteure ait fait l'effort de se conformer à une tradition littéraire pour la jeunesse ; au contraire, comme d'habitude, elle entraîne ses lecteurs dans des péripéties qui révèlent l'imperfection du quotidien. Puisque Bayard Éditions lui avait proposé d'écrire un roman pour le magazine Je bouquine, on pouvait en effet s'attendre à ce que l'écrivaine adapte sa poétique aux jeunes lecteurs en les ménageant quant à la complexité de l'histoire, mais c'est tout le contraire. Il s'agit même pour elle d'aller à rebours du genre qu'est le conte : «Comme je ne m'intéresse pas du tout au conte, je leur ai dit que j'allais raconter une histoire actuelle. » (Pfaff 116)

Inspiré d'un fait divers ${ }^{9}$, Haïti chérie est l'histoire d'une jeune adolescente de 13 ans, Rose-Aimée, qui se voit obligée de quitter ses parents pour aller travailler à Port-au-Prince sous les ordres d'une femme aisée. Or, la ville, décrite au départ comme un eldorado, va très vite se transformer en un calvaire pour la jeune

\footnotetext{
${ }^{8}$ Nous entendons ce terme, conformément à l'usage aujourd'hui le plus courant, dans un sens essentiellement chronologique, par différence avec postcolonial sans trait d'union, qui renvoie aux orientations politiques des théories postcoloniales, et plus généralement au « writing back». Post-colonial, dans ce sens socio-historique, peut néanmoins se référer, comme nous le verrons plus loin, à la postcolonie analysée par Achile Mbembe. La dimension proprement postcoloniale de l'œuvre de Maryse Condé relève d'une autre problématique, qui affleurera ci-dessous, notamment lorsqu'il sera question de la mémoire de l'esclavage.

${ }^{9}$ Ainsi que l'explique l'auteure elle-même, l'histoire d'Haïti chérie est « inspirée d'événements authentiques. Le 15 février 1980, on retrouvait sur les côtes de Floride (U.S.A) les corps d'une vingtaine d'Haïtiens. C'étaient en majorité des femmes et des enfants. Rose-Aimée aurait pu être parmi eux. Le capitaine du navire les avait forcés à se jeter à la mer. [...]» (Haïti chérie 60)
}

Alternative francophone

https://journals.library.ualberta.ca/af/index.php/af 
fille qui n'aura d'autre solution que celle d'embarquer clandestinement pour les États-Unis, aux côtés d'autres boat-people. Le texte nous décrit une société en pleine crise politique sous le règne de Duvalier, durant lequel les enfants en bas âge travaillent clandestinement pour aider leurs familles, sous la férule d'employeurs souvent très rigoureux. Lorsqu'ils sont abandonnés à eux-mêmes, ces enfants sont livrés à la rue où ils se débrouillent pour survivre et résister à l'insécurité et aux menaces nocturnes.

Au début du récit, la ville est perçue par tous les villageois comme un espace de réalisation ; les parents y voient une ascension sociale et les adolescents admirent le bien-être et les loisirs qu'on y trouve : « On dit qu'à Port-au-Prince, les maisons sont éclairées avec de l'électricité. Tu tournes un robinet et l'eau coule. Pas besoin de marcher des kilomètres sous le soleil avec un seau en équilibre sur la tête. Et puis, il y a le cinéma, la télévision. » (11). Or, dès son arrivée en ville, l'héroïne va connaître une série d'échecs correspondant à la structure même de la narration. Le premier correspond au deuxième chapitre dans lequel Rose-Aimée est plusieurs fois maltraitée et rudoyée par Madame Zéphyr, le deuxième est relaté dans le chapitre suivant, lorsqu'elle perd l'argent de cette dernière et se retrouve dans la rue ; le troisième, quant à lui, est celui du mauvais traitement que lui réserve Monsieur Modestin dans le quatrième chapitre, et le dernier (cinquième chapitre), enfin, n'est autre que le voyage qui n'aboutira jamais, étant donné que la jeune fille et son amie mourront, noyées, durant la traversée. Le roman est donc une suite d'insuccès : l'héroïne évolue dans un cercle vicieux où la résolution d'un obstacle conduit à une nouvelle épreuve, jusqu'à ce qu'elle atteigne l'épreuve ultime et fatale.

Le schéma est un peu le même dans Chiens fous dans la brousse, une auto-réécriture de Ségou en version jeunesse qui relate le rapt des enfants durant la période de l'esclavage. Ce roman historique est une autre commande de Bayard Éditions qui propose une quatrième fois à l'écrivaine de collaborer avec le magazine Je bouquine. Une fois de plus, Maryse Condé déjoue les attentes de son lectorat puisqu'elle ne raconte pas la vie dans les champs de cannes ou de coton. Comme dans Moi Tituba sorcière... Noire de Salem, elle évite l'esclavage en lui-même pour situer le récit à un autre moment de cette période historique, en Afrique ${ }^{10}$. Ainsi, plutôt que de traiter de la vie dans les champs de cannes à sucre, elle aborde la capture des enfants et se termine sur le sort de ces futurs esclaves dans le Nouveau Monde. L'histoire raconte comment deux jumeaux bambaras partis à la chasse aux lions avec leur père Tiéfolo se font enlever par des « chiens fous » (voleurs d'enfants) lors d'une fugue nocturne : Naba se pend avant d'être vendu à des marchands d'esclaves arabes, et Malobali est vendu à un médecin français chez qui il fera la rencontre d'Ayodele, fille de Mansa, elle aussi enlevée par les « chiens fous » lors d'une escapade hors des murs du palais de son père.

Comme dans l'exemple précédent, l'échec semble déterminer l'orientation du récit. Dès l'incipit, les enfants sont présentés par la voix narrative comme des antihéros : destinés à être des chasseurs de lions, comme leur père Tiéfolo, les jumeaux dérogent à un lignage héroïque, n'ayant d'yeux que pour la poésie et le chant, un choix qui sans doute les conduira vers leur propre malheur. Quant à Ayodele, elle a, elle aussi, un vilain défaut : la convoitise du monde extérieur qu'elle rêve de voir malgré les multiples interdictions parentales. Ainsi, comme la chèvre de Monsieur Seguin qui se fait dévorer par le loup durant une fugue, les enfants du roman bravent les interdictions plusieurs fois formulées à leur endroit. Les conséquences s'avèrent tout aussi désastreuses puisque, échouant dans les quêtes qu'ils se fixent euxmêmes, ils ne réussissent qu'à se faire enlever, chagrinant ainsi leurs parents.

\footnotetext{
${ }^{10}$ Cet aspect a fait l'objet d'un chapitre d'ouvrage coécrit avec Pauline Franchini intitulé « Écrire l'esclavage pour les adultes et pour les jeunes : Maryse Condé et les enjeux de la mixité éditoriale », Écrire l'esclavage dans la littérature pour la jeunesse.
} 
Le dernier roman, La Belle et la bête : une version guadeloupéenne, s'inspire, d'une manière tout à fait assumée comme en témoigne ce titre, du célèbre conte La Belle et la Bête dont on doit le succès littéraire à la version de Madame Leprince de Beaumont. Outre quelques thématiques communes (l'amour et la beauté), les deux textes se différencient par le contexte géographique et culturel, ainsi que par la dimension réaliste : tandis que le conte de Beaumont a une allure de conte de fées, Maryse Condé choisit une tonalité pathétique. Contrairement aux personnages de Madame Leprince de Beaumont qui se caractérisent par la pureté du cœur et de l'âme, la version condéenne entraîne son lecteur sous les tropiques caribéens, avec une Belle avare et égoïste qui épouse Carmélien la Bête pour des raisons uniquement financières, sans éprouver le moindre sentiment pour ce dernier. Comme dans les précédents exemples, l'histoire s'achève sur un échec : celui du mariage entre les deux protagonistes, celui des identités - Bella rejetée par la famille italienne de son père - et celui du désenchantement de Bella face à l'aisance, puisqu'elle va finalement s'ennuyer dans cette nouvelle vie confortable. D'ailleurs l'écrivaine reprend ironiquement un élément du happy end des contes de fées pour en faire un échec : Bella est enceinte de Carmélien, mais cette grossesse n'annonce pas des jours meilleurs : « Au contraire de Carmélien qui exultait de bonheur, cela ne lui procura aucune joie, aucun sentiment de fierté. Qu'en serait-il de cet enfant ? Que lui viendrait-il en partage? Aurait-il le physique de son père et l'appelleraiton aussi la "Bête" pour ensuite le couvrir d'éloges? Aurait-il son don incomparable? » (91).

Dans les trois romans susmentionnés, les schémas narratifs mettent en scène des antihéros face aux conséquences de leurs mauvaises actions. La voix narrative choisit de responsabiliser les enfants personnages, confirmant ainsi les propos suivants de Véronique Chelin à propos de Le coeur à rire et à pleurer : contes vrais de mon enfance : «l'enfance [chez Condé] n'apparait pas du tout comme un monde isolé, protégé ou édénique. Au contraire, l'univers complexe des adultes pénètre celui, plus intime, de [ce dernier]. » (52). Ainsi, les échecs auxquels ils sont confrontés sont généralement dus à leurs mauvais comportements; par exemple lorsque la petite Ayodele se fait kidnapper, les passants feignent de ne rien voir : «Après tout, une petite fille qui fait une escapade et se balade seule ne récolte que ce qu'elle mérite. » (44). On peut, bien que l'auteure refuse à ses romans une dimension moraliste, relever une sorte d'appel à la prise de conscience des adolescents, un moment de la vie où les jeunes sont en quête d'identité et d'indépendance. Comme dans le conte d'avertissement, Maryse Condé met en garde ses jeunes lecteurs ; par exemple, La Belle et la Bête se clôt sur un propos que l'on peut apparenter à la morale de l'histoire : «Finalement qu'importe! La vie est une mégère acariâtre qui boite des deux pieds. Les dons qu'elle accorde ne font jamais recette. La Belle peut devenir la Bête et vice versa. » (91). Cela constitue une divergence avec la littérature pour les adultes qui se passe de cette dimension moraliste, au contraire du corpus pour la jeunesse où elle est clairement énoncée, même si on peut y voir aussi une dimension politique.

On remarque donc que les structures narratives des œuvres de Condé portent en elles-mêmes les traces d'une " pédagogisation », puisque l'évolution du récit repose généralement sur les actes qui emmènent les protagonistes à leur chute et donc à la prise de conscience du lecteur, susceptible de réaliser les conséquences des comportements des personnages. Toutefois, il faut nécessairement préciser que certains échecs présentent une dimension positive, comme c'est le cas avec la mort de Naba ou celle de RoseAimée dont la description poétique masque le caractère tragique de la scène. Concernant les fins des récits, on retient une ambiguïté et une incertitude à propos du destin des protagonistes, «Condé ne promet aucune fin heureuse à ses [lecteurs]. De fait, ses récits ne se referment ni sur un triomphe ni sur des hurlements de peur. » (Caute 69-70). La description du départ de Malobali et d'Ayodele pour le Nouveau

Alternative francophone

https://journals.library.ualberta.ca/af/index.php/af 
Monde, ainsi que la grossesse de Belle, invitent le lecteur à réfléchir à un futur incertain qu'il ne connaîtra jamais, le sort des personnages étant laissé à son imagination.

\section{CONTEXTES SOCIO-POLITIQUES EN CRISE}

Les œuvres de Maryse Condé sont réalistes et mettent généralement en avant les difficultés engendrées par les régimes politiques post-coloniaux. C'est d'ailleurs ce qui fait, de sa littérature de jeunesse, une littérature postcoloniale au même titre que sa littérature pour les adultes. L'auteure y aborde en effet des thématiques tout aussi complexes, comme la dictature, l'immigration, la guerre ethnique, les violences politiques et historiques. Dans un article intitulé « Images de l'injustice et de la violence dans Haïti chérie et $A$ la courbe du Joliba de Maryse Condé », Adeline Caute remarque que : « les héroïnes de Condé sont assujetties à des violences multiples en raison de catégories d'identité qu'elles ne peuvent pas changer, telle que la couleur de leur peau ou leur genre. » (69). Or, ce constat vaut pour la majorité des œuvres de l'auteure, peu importe le lecteur à qui elle s'adresse. Dans Desirada, Marie-Noëlle est dès son bas âge victime des injustices sociales, ainsi qu'Ivan et Ivana dans Le fabuleux et triste destin d'Ivan et Ivana, qui évoluent dans des sociétés marquées soit par l'Histoire de l'esclavage et ses conséquences sur la sociabilité, soit par l'échec de l'État moderne. Qu'il s'agisse donc des œuvres qui ont déjà été citées ou d'autres textes comme Victor et les barricades, les jeunes personnages principaux évoluent toujours dans un contexte socio-politique déterminé par l'Histoire. Dès lors, la poétique de l'échec réside aussi bien dans la référentialité des œuvres.

En lisant Haïti chérie ou Victor et les barricades, le lecteur est confronté à plusieurs scènes violentes. Dans le premier texte, Rose-Aimée est tour à tour injuriée par ses patrons et les illustrations colorées de Marcelino Truong accentuent la gravité de la situation, puisqu' on y voit Rose-Aimée en train de se faire battre par Madame Zéphyr (20). De même, les dernières images du récit, dans lesquelles on voit des visages effrayés (59), sont susceptibles de déconcerter le jeune lecteur. Le second texte, quant à lui, présente, outre une rupture brutale entre Victor et sa mère, plusieurs scènes de violence : le jeune adolescent se retrouve face à des émeutes politiques. Cette sorte de « brutalisme » (Mbembe 2020) est davantage lisible dans les images de Marcelino Truong représentant des personnages qui s'affrontent avec des armes blanches (59); d'ailleurs, la page de couverture du magazine montre des jeunes personnages échappant aux violences policières avec, en arrière-plan, des C.R.S armés de fusils et de matraques. Durant une scène de violence, Victor reçoit un coup sur la tête et, là encore, comme dans les précédents textes, la voix narrative responsabilise le jeune protagoniste en recourant à un discours indirect libre qui se situe entre la voix du narrateur et celle du personnage : "C'était sa punition pour avoir été si paresseux en classe, pour avoir baillé sur ses leçons et bâclé ses devoirs. » (56-58). Et, pareil aux adultes insensibles au kidnapping d'Ayodele, « un groupe passa en courant sans même tourner la tête vers lui, étendu là sur le trottoir. Sur un balcon, un homme sortit pour le regarder au contraire. Puis retourna à l'intérieur de la maison dont il referma la porte. » (58). L'auteure choisit de mettre les personnages face à leurs destins; l'inaction des grandes personnes vis-à-vis des enfants en difficulté propose aux jeunes lecteurs un point de vue peu réconfortant sur une société dans laquelle ils seront les premiers comptables de leur destinée.

Les crises sociétales décrites dans les romans ne sont que les résultats des échecs politiques qui caractérisent les «postcolonies $»^{11}$. En effet, les jeunes personnages évoluent dans des contextes en crise :

\footnotetext{
${ }^{11}$ Selon Achille Mbembe, il s'agit des « sociétés récemment sorties de l'expérience que fut la colonisation, celle-ci devant être considérée comme une relation de violence par excellence. Mais plus que cela, la postcolonie est une pluralité chaotique, pourvue d'une cohérence interne, de systèmes de signes bien à elles, de manières propres de fabriquer des simulacres ou de construire des stéréotypes, d'un art spécifique de la démesure, de manières particulières d'exproprier le sujet de ses identités. [...]» (Mbembe 138-140).
} 
Rose-Aimée subit les affres d'une dictature politique, Victor est confronté aux problèmes raciaux, et Aïcha et ses sœurs jumelles sont obligées de partir de la Côte-d'Ivoire pour échapper à la guerre ethnique qui y sévit. La dimension historique des récits confirme davantage cette critique post-coloniale lisible à partir de la poétique de l'échec. Si la société haïtienne est sous un régime dictatorial, les malheurs de Rose-Aimée sont également dus à la mauvaise foi de ses employeurs ; la description de ces derniers (mulâtres) fait penser à la hiérarchie raciale en Guadeloupe, et les rapports qu'ils entretiennent avec la jeune fille ressemblent beaucoup à la situation des esclaves dans les plantations : « ... Rose-Aimée agenouillée sur le sol s'apprêtait à attirer vers elle le seau d'eau mousseuse, d'un coup de pied monsieur Modestin l'envoya valser à l'autre bout de la pièce. » (44-45).

Plus loin dans la narration, l'écrivaine elle-même fait resurgir l'Histoire de l'esclavage en comparant la réaction de Rose-Aimée à celle d'un esclave révolté : «Comme si l'esprit de ses ancêtres africains, qui avaient conquis leur liberté en battant les puissantes armées envoyées par Bonaparte, la possédait à nouveau. À sa manière, elle revivait le combat de Makandal, de Boukman... » (45) À propos de ce même passage, on ne peut ignorer l'appel de note qui explique au lecteur l'identité de Makandal et de Boukman, une pratique littéraire de nature didactique, dont le but est de faciliter la compréhension du jeune lecteur en élargissant par là-même sa culture générale.

Cette même question est abordée dans Victor et les barricades dont l'intrigue repose sur un conflit racial. L'écrivaine n'hésite pas à y critiquer l'institution académique dont le programme éducatif ne permet pas aux enfants de s'informer sur leur Histoire. Maryse Condé désapprouve en effet le système scolaire auquel elle fut soumise ${ }^{12}$ durant son enfance, et semble s'exprimer par la voix de ses protagonistes. À l'image de la jeune Maryse, la voix narrative exprime l'insatisfaction de Victor face aux contenus des cours :

Pourquoi était-il toujours le dernier en toutes les matières ? Pourquoi ne pouvait-il rien retenir ? Ces problèmes où il était toujours question de trains qui allaient, venaient, se croisaient alors qu'il n'avait jamais vu de trains... [...] Pourquoi ne lui parlait-on pas de ce qui l'entourait ? Pourquoi ne lui apprenaiton pas à nommer les arbres majestueux, les arbustes, les fleurs aux parfums violents, les poissons aux écailles de laque bleu, jaune ou rose de sa petite Guadeloupe ? (18)

D'ailleurs, n'est-ce pas l'une des raisons pour lesquelles l'auteure écrit pour le jeune public ? Dans un entretien avec Cécile Lebon, elle confie : « Aujourd'hui plus que jamais il est bon d'intéresser les jeunes caribéens à l'histoire coloniale. Les migrations, la globalisation non élucidée, ajoutent à la complexité du monde. Il est bon par exemple d'expliquer que ce dernier phénomène dont on parle tant n'est pas nouveau pour la diaspora africaine. » (11). Il semble aussi que c'est à ce projet que répond Chiens fous dans la brousse puisqu'au lieu de parler d'esclavage, le récit explique plutôt aux enfants comment certains Africains se sont retrouvés hors de leur continent d'origine.

Enfin, l'échec politique est lisible dans ce qu'Amadou Kourouma nomme ironiquement « Les soleils des indépendances », à savoir les régimes politiques africains après la colonisation. L'album À la courbe du Joliba revient sur la mauvaise gestion des États, qui a souvent conduit aux guerres ethniques comme c'est le cas dans l'extrait suivant : «selon lui, leur groupe ethnique, les Mandés, las d'être traité en paria, ne supportait plus le pouvoir central d'Abidjan. Il menaçait de créer son propre État. Déjà les commandos

${ }^{12}$ Dans Le coeur à rire et à pleurer : contes vrais de mon enfance, Maryse Condé raconte comment elle a découvert l'histoire de l'esclavage (voir les chapitres : « Leçon d'Histoire » et « Chemin d'école »).

Alternative francophone

https://journals.library.ualberta.ca/af/index.php/af 
s'opposaient à l'armée nationale et occupaient les villes. Déjà le sang avait coulé. » (10). Ainsi, dès le début du récit, le lecteur est plongé dans une société en proie aux conflits politiques, constatant les conséquences de la guerre sur la famille et sur la population en général. Dans le texte, un autre échec est aussi mis en avant ; il s'agit des découpages territoriaux dont nous savons qu'ils ont été opérés par l'administration coloniale, mais le narrateur en fait une question plus générale : «C'était donc ça le Mali ? [...] Ce n'était guère différent du nord de la Côte-d'Ivoire. À peine plus sec. À peine plus roux. À peine plus chaud. Les mêmes cases en banco. [...] Quelle main a décidé de tracer les frontières ? Peut-on imaginer un monde où elles n'existeraient pas?» (25).

\section{CONCLUSION}

Toutes ces mises en scène de l'échec, bien qu'elles diffèrent selon les œuvres (thème, narration, esthétique), confirment le réalisme de l'écriture condéenne. La fiction permet ainsi à l'écrivaine de servir aux adolescents une critique sociale et historique en responsabilisant les jeunes personnages. Néanmoins, il ne s'agit pas non plus d'imposer une leçon inspirée par l'opinion dominante : ainsi que le constate Noëlle Carruggi, elle « résiste à l'univocité et présente au lecteur un certain degré d'aspérité : refus de s'aligner à un mouvement littéraire, recherche incessante de nouvelles formes narratives propres à révéler un aspect ignoré de notre rapport au monde et de l'adéquation de l'être à sa vérité intérieure. » (10). Cette orientation ne va pas de soi en littérature de jeunesse, où de nombreux écrivains s'abstiennent de décrire des univers aussi durs ${ }^{13}$. Cet aspect n'empêche cependant pas le succès auprès des enfants : Haïti chérie, dont le réalisme inquiétait fortement l'éditeur ${ }^{14}$, « est un livre qui a beaucoup circulé dans les écoles et qui a bien marché en tant que livre d'enfant. » (Pfaff 119). Dès lors, on ne peut pas penser que la dureté en question aurait un impact négatif sur la réception de cette littérature. On peut même faire l'hypothèse qu'elle faciliterait sa réception parce qu'elle répond aux reproches bien connus de mièvrerie et de moralisme facile.

Finalement, si on ne peut pas affirmer que la visibilité relativement faible de la littérature pour la jeunesse de Maryse Condé est tributaire de la mise en scène de l'échec dans ses œuvres concernées, la critique gagnerait néanmoins à changer le regard qu'elle porte sur celles-ci.

D’une manière générale, cette approche des œuvres de Maryse Condé pour la jeunesse montre encore une fois que « la littérature d'enfance et de jeunesse ne doit pas être considérée comme "une sous-littérature "pour adultes"' qui vient peu ou prou s'opposer à la littérature légitime. Elle se présente plutôt comme un modèle réduit de ce que nous connaissons dans le champ des lectures d'adultes, faisant cohabiter littérature légitime, production faiblement qualifiée et éphémère marchandise. » (Nières-Chevrel 112113). Certes, la littérature de jeunesse est un secteur littéraire spécifique, où les modalités éditoriales et littéraires sont différentes parce qu'elle prend en compte les goûts, ainsi que les capacités intellectuelles et culturelles d'un destinataire particulier; dès lors, elle nécessite, d'une part, des outils d'analyse singuliers autant dans la réception que dans la critique, et, d'autre part, des politiques du livre et de lecture à même de lui conférer une place au sein du patrimoine culturel et académique général. Mais cette spécificité n'empêche pas qu'elle fait partie du même monde que celui de la littérature générale et que, dans le cas de Maryse Condé, les deux corpus donnent à lire ce même monde.

\footnotetext{
${ }^{13}$ Dans le même sens que l'on a parlé aussi des « romans durs » de Georges Simenon.

${ }^{14}$ Dans son entretien avec Françoise Pfaff, Maryse Condé explique l'hésitation des éditions Bayard en ces termes : « c'est la maison d'édition Bayard qui m'a demandé une histoire pour enfants pour sa collection Je bouquine.

Comme je ne m'intéresse pas du tout aux contes, je leur ai dit que j'allais raconter une histoire très actuelle. Ils ont commencé par tiquer en me disant qu'on ne pouvait pas raconter une histoire très actuelle à des enfants de onze ou douze ans. Puis quand je leur ai donné le manuscrit, ils n'ont rien trouvé à redire. Mais ils persistaient à croire que ce n'était pas vraiment une histoire pour les enfants. [...] Ils voulaient donc changer la fin. » (Pfaff 116-117).
} 


\section{BIBLIOGRAPHIE}

Amedegnato, Ozouf Sénamin et al. « Avant-propos », Légitimité, légitimation, édité par Ozouf Sénamin Amedegnato et al., Presses universitaires de Bordeaux, 2011, pp. 11-18.

Attikpoé, Kodjo. « L'émergence de la littérature et de l'édition d'enfance et de jeunesse en Afrique francophone : l'apport féminin », L'édition de jeunesse francophone face à la mondialisation, édité par Jean Foucault et al. L’Harmattan, 2010, pp. 151-160.

Bertiau, Christophe. «Introduction ». Autopsie de l'échec littéraire, édité par Christophe Bertiau et Chanel de Halleux, COnTEXTES, no. 27, 2020, http://journals.openedition.org/contextes/9173.

Carruggi, Noëlle, éditrice. Maryse Condé : Rébellion et transgressions. Karthala, 2010.

Caute, Adeline. «Images de l'injustice et de la révolte dans Haïti chérie et À la courbe du Joliba de Maryse Condé », Les pouvoirs de la littérature de jeunesse, édité par Kodjo Attikpoé, Peter Lang, 2018, pp. 59-71.

Chelin, Véronique. "Le récit d'enfance au féminin : les cas de Condé, Fidji et Patel », Les écrits contemporains de femmes de l'Océan Indien et des Caraïbes, édité par Rohini Bannerjee et Karin Schwerdtner, Les cahiers du GRELCEF, 2012, pp. 49-65.

Condé, Maryse. Le fabuleux et triste destin d'Ivan et Ivana. Robert Laffont, 2017.

—. Le cour à rire et à pleurer : contes vrais de mon enfance. Didier, 2013.

—. La Belle et la Bête : une version guadeloupéenne, Larousse, 2013.

—. À la courbe du Joliba, illustré par Letizia Galli. Grasset Jeunesse, 2006.

—. Chiens fous dans la brousse, illustré par Christel Espié. Je bouquine, no. 268, juin 2006.

—. Désirada. Robert Laffont, 1997.

—. Victor et les barricades, illustré par. Marcelino Truong. Je bouquine, no. 61, mars 1989.

—. Haïti chérie, illustré par Marcelino Truong. Je bouquine, no. 39, mai 1987.

Condé, Maryse. Entretien réalisé par Cécile Lebon, Takam Tikou : la revue des livres pour enfants, no. $11,2004$.

Delbrassine, Daniel. «Des romanciers "pour la jeunesse" qui ne le restent pas : circulation des auteurs entre le champ de la littérature de jeunesse et celui de la littérature générale », L'édition de jeunesse francophone face à la mondialisation, édité par Jean Foucault et al. L'Harmattan, 2010, pp. 161-173.

Fort, Pierre-Louis. «Littérature de jeunesse et esclavage : Chiens fous dans la brousse de Maryse Condé ", Esclavage et littérature de jeunesse, édité par Christiane Chaulet Achour, Classiques Garnier, 2016, pp. 115-126.

Alternative francophone

https://journals.library.ualberta.ca/af/index.php/af 
Franchini, Pauline ; Mouloungui, Merveilles L. «Écrire l'esclavage pour les adultes et pour les jeunes : Maryse Condé et les enjeux d'une mixité éditoriale », Écrire l'esclavage dans la littérature pour la jeunesse, édité par Christiane Connan-Pintado et al., Presses universitaires de Bordeaux, 2021, pp. 61-73.

Franchini, Pauline. «De Ségou à Chiens fous dans la brousse : un exemple d'autoréécriture chez Maryse Condé », Écrire pour la jeunesse et pour les adultes : d'un lectorat à un autre, édité par Marion Mas et Anne-Marie Mercier-Faivre, Classiques Garnier, 2020, pp. 163-178.

Hunt, Peter. «Introduction : The World of Children's Literature Studies », Understanding Children's literature, édité par Peter Hunt, Routledge, 1999, pp. 1-14.

Jeanne, Max. «Quelques éléments sur la Caraïbe française : Guadeloupe. [Entretien avec Max Jeanne] », Situations de l'édition francophone d'enfance et de jeunesse, édité par Luc Pinhas, L'Harmattan, 2008, pp. 333-336.

Mbembe, Achille. De La Postcolonie : essai sur l'imagination politique dans l'Afrique contemporaine. Karthala, 2000.

Nières-Chevrel, Isabelle ; Perrot, Jean. Éditeurs. Le dictionnaire du livre de jeunesse : la littérature d'enfance et de jeunesse en France. Éditions du Cercle de la Librairie, 2013.

Nières-Chevrel, Isabelle. «Faire une place à la littérature de jeunesse », Revue d'histoire littéraire de la France, vol. 102, no. 1, 2002, https://www.cairn.info/revue-d-histoire-litteraire-de-la-france2002-1-page-97.htm.

Nièvres-Chevrel, Isabelle. Introduction à la littérature de jeunesse. Didier Jeunesse, 2009.

Perrot, Jean. Mondialisation et littérature de jeunesse. Éditions du Cercle de la librairie, 2008.

Pfaff, Françoise. Entretiens avec Maryse Condé. Karthala, 1993.

Pinhas, Luc, éditeur. Situations de l'édition francophone d'enfance et de jeunesse. L'Harmattan, 2008.

Prince, Nathalie. «Introduction », La littérature de jeunesse en question(s), édité par Nathalie Prince. Presses universitaires de Rennes, 2009, pp. 9-26. 